\title{
Classification of EEG Signal by Using Optimized Quantum Neural Network
}

\author{
Dalael Saad Abdul-Zahra ${ }^{\text {*, }}$, Ali Talib Jawad ${ }^{2}$, Hassan Muwafaq Gheni ${ }^{3}$, Ali Najim Abdullah ${ }^{4}$ \\ ${ }^{1}$ Dept. of Medical Physics Hilla University College Babylon, Iraq \\ ${ }^{2}$ Dept. of Medical Instrumentation Technologies Engineering Hilla University College Babylon, Iraq \\ ${ }^{3}$ Computer Techniques Engineering Department, Al-Mustaqbal University college, Hillah 51001, Iraq \\ ${ }^{4}$ Dept. of Medical Instrumentation Technologies Engineering Hilla University College Babylon, Iraq
}

\begin{tabular}{l} 
Article Info \\
\hline Article history: \\
Received Nov 1, 2021 \\
Revised Dec 16, 2021 \\
Accepted Dec 23, 2021 \\
\hline Keywords: \\
Quantum Neural Network \\
Wavelet \\
EEG \\
PSO
\end{tabular}

\begin{abstract}
In recent years the algorithms of machine learning were used for brain signals identification as a useful technique for diagnosing diseases like Alzheimer's and epilepsy. In this paper, the Electroencephalogram (EEG) signals are classified using an optimized Quantum neural network (QNN) after normalizing these signals. The wavelet transform (WT) and the independent component analysis (ICA) were utilized for feature extraction. These algorithms were used to reduce the dimensions of the data, which is an input to the optimized QNN for the purpose of performing the classification process after the feature extraction process. This research uses an optimized QNN, a form of feedforward neural network (FFNN), to recognize the EEG signals. The Particle swarm optimization (PSO) algorithm was used to optimize the quantum neural network, which improved the training process of the system's performance. The optimized QNN provided us with somewhat faster and more realistic results. According to simulation results, the total classification for ICA is 82.4 percent, while the total classification for WT is 78.43 percent; from these results, using the ICA for feature extraction is better than using WT.
\end{abstract}

Copyright (C) 2019 Institute of Advanced Engineering and Science. All rights reserved.

\section{Corresponding Author:}

Dalael Saad Abdul-Zahra,

Dept. of Medical Physics Hilla University College Babylon, Iraq.

Hilla University College ,Babylon, Iraq.

Email: dalael20175@gmail.com

\section{INTRODUCTION}

Electroencephalogram (EEG) records the electrical potentials produced by the brain. EEG activity analysis has mostly been utilized in clinical settings to diagnose pathologies and epilepsies since Hans Berger's recording of rhythmic electrical activity from the human scalp[1]. With the progress of computers and related technologies, it is now possible to measure EEG changes using a variety of methods[2]. The EEG signals are exceedingly complex for an inexperienced to be observed to interpret when compared to other biological signals, involves the spatial mapping of functions onto various parts of the brain, as well as the implantation of electrodes [3]. As in reaction recordings, data processing can include determining a restricted feature set that only includes the data needed for quantification, or features extraction and subsequent pattern recognition[4]. In this paper, the optimized Quantum neural network (QNN) which is used for EEG signal classification. The quantum neural networks are optimized by using the Particle Swarm Optimization (PSO), which improved the QNN training process[5]. For the feature extraction process, we use two approaches for feature extraction and comparing with the results; one of them is the Independent Components Analysis (ICA), a technique for feature extracting the data from a set of data, and the other is wavelet transform[6]. Each extracted signal is statistically independent of all other extracted signals, which is a distinguishing feature extracted signals[7]. The basis signal used for decomposing the signal produces a time and scale distribution rather than "frequency" information in the traditional sense. The wavelet is stretched or compressed by a factor of two when the scale is changed. As a result, every signal can be reconstructed using a single wavelet or ICA as the foundation and as many ICA or wavelets as needed at various scales and times[8]. 
This paper is organized to present some sections as follows. Section 2 presents the research methodology that presents the methods implemented for this work and the recognition system used to classify the EEG signals and the calculations for the feature extraction by using ICA and wavelet and the classification by using the optimized quantum neural networks. Section 3 is the simulation results for the EEG. Section 4 provides discussion and finally section 5 offers the conclusions.

\section{Literature survey}

The previous studies by Ömer Türk, Mehmet Siraç [9] in 2021 revealed that EEG signals are presented as a method for detecting emotional states. We intend to classify EEG data using the suggested method without using any pre-processing transforms (Fourier transform, wavelet transform, etc.). As classifiers, convolutional neural networks (CNNs) and the seeds of the EEG dataset, that contain a three distinct emotional states a positive, negative, and neutral, are used. The records used were collected throughout three sessions from 15 participants. channel-time in its natural state. Without any processing, the records of EEG are converted into $28 \times 28$ segment. The three emotions performance averages of all participants were found to be 88.84 percent as a consequence of the classification. According to the participants, the highest categorization performance is 93.91 percent, while the classification performance lowest is 77.70 percent. Compared with our work, the PSO algorithm improves the quantum neural network performance and gives a brilliant result; also, using the ICA as a feature extraction makes high system performance as compared with a wavelet approach. Other research by H. Donmez and N. Ozkurt [10] in 2019 in machine perception shows that emotion recognition is a critical task for computers. As a result, in this reserch, the participant is shown as a comprising video amusing, scary, and sad snippets while their EEG signals are evaluated using a EEG Headset Neurosky Mindwave. CNN is given a spectrogram of EEG signal, and the images of brain signal spectrograms are used to classify three emotions. By comparing with our paper, the performance of the system is better because the PSO algorithm optimized the quantum neural network training process, and the performance is improved.

\section{RESEARCH METHOD}

\subsection{Methods forThe Feature Extraction}

\subsubsection{Wavelet Transorm}

If a signal does not change considerably over time, it is considered to be stationary. The stationary signals can be subjected to the Fourier transform [11]. However, many signals, like EEG, can have nonstationary or transient features. As a result, applying the Fourier transform directly to such signals is not recommended [12].The approaches of the time frequency, like the wavelet transform, should be utilized in this case. A diffrent of probing functions can be utilized in wavelet analysis. The continuous wavelet transform's defining equation is derived from this concept (CWT).

$$
\mathrm{W}(\mathrm{a}, \mathrm{b})=\int_{-\infty}^{\infty} x(t) \frac{1}{\sqrt{a}} \varphi\left(\frac{t-b}{a}\right) d t
$$

Where the variable $a$ modifies the function over $x(t)$ and variable $b$ modifies the temporal scale of the probing function $w$. The wavelet function, $\varphi$, is stretched down the time axis if an is larger than one, and it touches the function if an is less than one (but still positive)[13]. The probing function $w$ can be any of a variety of functions, but it always takes on an oscillating form, hence the word "wavelet." For all values of a. and the term $\frac{1}{\sqrt{a}}$ is the normalization factor which ensures the energy is the same [14]. In DWT signal analysis, choosing the right wavelet and the right number of layers of decomposition is crucial [15]. The signal's principal frequency components influence the number of stages of decomposition. The wavelet coefficients are changed so that those components of the signal that correlate well with the frequencies required for signal categorization are retained [16]. Because there are no meaningful frequency components in EEG signals above $30 \mathrm{~Hz}$, the number of levels was set to 5. As a result, the signal is divided into D1-D5 details and a final approximation.

\subsubsection{Independent component analysis}

Using the ICA, feature extraction based on a multivariate random signal is turned into a signal with mutually independent components. From mixed signals, this approach can be utilized to isolate independent components. In this context, independence refers to the fact that the information that carried by the one component cannot be deduced from information carried by the others. This means that the combined independent probability of the values is estimated as a product of their individual probabilities in statistical terms [17].

Suppose that $\mathrm{c}$ is the a scalar source signals $\mathrm{x}_{\mathrm{i}}(\mathrm{t})$ for $\mathrm{i}=1, \ldots, \mathrm{c}$, where $\mathrm{t}$ is a time index of $1 \leq \mathrm{t} \leq \mathrm{T}$. We combined the $\mathrm{c}$ values at a given time into the vector $\mathrm{x}(\mathrm{t})$ for notational simplicity and assumed the vector has a zero mean. We may write the multivariate density function because of our independence assumption and the assumption of no noise:

$$
B(x(t))=\prod_{i=1}^{c} B\left(x_{i}(t)\right)
$$


Assume that at each moment, a d-dimensional data vector is .

$$
\mathrm{Y}(\mathrm{t})=\mathrm{A} \times(\mathrm{t})
$$

Where $A$ is a c $x$ d scalar matrix, and we'll need $d \geq c$. The Independent component analysis is used to reconstruct the source signals from detected signals. We're looking for an actual matrix W that looks like this:

$$
\mathrm{z}(\mathrm{t})=\mathrm{WY}(\mathrm{t})=\mathrm{Wax}(\mathrm{t})
$$

Where $\mathrm{z}$ denotes an estimated at the sources $\mathrm{x}(\mathrm{t})$, we're looking for $\mathrm{W}=\mathrm{A}^{-1}$, but neither A nor its inverse exist.We use maximum-likelihood approaches to figure out what $\mathrm{A}$ is. We employ a density estimate parameter by a $B^{\wedge}(\mathrm{Y}, \mathrm{A})$ and look for a vector parameters that minimized a difference between the estimated and source distributions[18].

\subsection{Classification Process by Optimized QNN}

The proposed system's main purpose is to analyze EEG data using ICA and wavelet transforms as an alternative technique to extract signal features, and to use an efficient optimized QNN for classification. Each EEG signal is made up of 2500 samples, each representing a vector pattern. The data set's vectors will be normalized. Knots are recovered after normalization, and the best knot is chosen to represent the essential characteristics of EEG signal shape and its specific points [19]. The feature vectors will be formed and then applied to the optimized QNN for classification or training. A three-layer (L) QNN is employed as an EEG signal classifier, with the retrieved features used for training and testing. The training process was repeated until the maximum number of iterations was reached. The output layer of QNN has (5) output neurons (no), and the input layer has 40 input neurons (ni). A scalar variable represents each signal, a vector of variables represents a collection of signals, and the processing for generating signals mixes from signal from the source using a sets of the mixing coefficients. The ICA shows how to represent a group of signals as a scattergram, with each point corresponding to the signals' values at a certain time, and how to apply a geometric transformation to each point using a set of mixing coefficients[20].

$$
\begin{aligned}
& x_{1}=a s_{1}+b s_{2} \\
& x_{2}=c s_{2}+d s_{2}
\end{aligned}
$$

Where the coefficients $\mathrm{a}, \mathrm{b}, \mathrm{c}$ and $\mathrm{d}$ sets of the mixing coefficients.

The resulting "mixing" points that are added can be combined with the original "source" points. To construct "signal" points, unmixing coefficients are utilized, which reversed the original signal's effect. The signal to signal source mixtures are transformed geometrically[21].

$$
\begin{aligned}
& s_{1}=\alpha_{1} x_{1}+\beta_{1} x_{2} \\
& s_{2}=\alpha_{2} x_{1}+\beta_{2} x_{2}
\end{aligned}
$$

Where $\alpha_{1}, \alpha_{2}, \beta_{1}$ and $\beta_{2}$ are unmixing coefficients set while $\mathrm{s}_{1}, \mathrm{~s}_{2}$ represents original signals. A signal x's discrete wavelet (DWT) which is obtained by running it through filters [22]. Following that, the samples were run through a low pass filter with a $\mathrm{g}$ impulse response; the two are convolutional:

$$
y[n]=(x * g)[n]=\sum_{t=-\infty}^{\infty} x[t] g[n-t]
$$

At the same time, a high-pass filter $\mathrm{h}$ is utilized to deconstruct the signal. The approximation coefficients and detail coefficients (from the high-pass filter) are outputs (from the lowpass) [23]. The fact that the two filters are connected is crucial, as they are referred to as a quadrature's mirrors filter. According to Nyquist's rule, half of the samples can now be discarded because half of the signal's frequencies have been deleted[10]. Following that, the outputs of the filter are subsampled by two g- high pass and h- low pass:

$$
\begin{gathered}
y_{L}=\sum_{t=-\infty}^{\infty} x[t] g[2 n-t] \\
y_{H}=\sum_{t=-\infty}^{\infty} x[t] h[(2 n+1)-t]
\end{gathered}
$$

Where $y_{L}$ and $y_{H}$ represent the outputs in $(g)$ high pass and (h) low pass sequentially. Because only half of each filter's output is used to separate the signal, this decomposition reduces the time resolution by half. However, each output frequency range is half that of the input [24]. The frequency accuracy has been increased by a factor of two.

With the down sampling operator $\downarrow$ :

$$
(y \downarrow t)[n]=y[t n]
$$

The preceding summary might be written in a simpler manner.

$$
\begin{gathered}
y_{L}=(x * g) \downarrow 2 \\
y_{L}=(x * h) \downarrow 2
\end{gathered}
$$


Calculating a total $\left(\mathrm{x}^{*} \mathrm{~g}\right)$ convolution with post-reduction, on the other hand, would be a waste of time [12]. Where these two arithmetic operations occur, the levitation diagram is optimal [25] .The classification approach was utilized to identify a best characteristics from signals that were deal with WT, ICA. As a result, the individual within a class scattering matrices and total within-class scattering matrices are represented as follows:

$$
\begin{aligned}
& S_{i}=\sum_{x \in X}\left(x-m_{i}\right)\left(x-m_{i}\right)^{T} \quad i=1,2, \ldots \\
& S_{\omega}=S_{1}+S_{2}
\end{aligned}
$$

Where $m_{i}$ represents vector of $i$-th class. Using Fisher criteria function and the Lagrange multiplier approach, we can find the transform vector $\mathrm{w}$ with the maximum between-class distance and the smallest within-class variance:

$$
\omega=S_{\omega}^{-1}\left(m_{1}-m_{2}\right)
$$

Each hidden node represents a file in the QNN, which inputs and one hidden layer of nh nodes. There are no output units in a multilevel function (equal to 15). Sigmoidal output units. The output equation for the hidden layer is:

$$
\begin{gathered}
h_{j . k}=\frac{1}{n_{s}} \sum_{r=1}^{n_{s}} h_{j . k}^{r}=\frac{1}{n_{s}} \sum_{r=1}^{n_{s}} \operatorname{sgm}\left(\beta_{h}\left(h_{j . k}^{r}-\theta_{j}^{r}\right)\right) \\
\operatorname{sgm}(\tau)=\frac{1}{(1+\exp (-\tau))}
\end{gathered}
$$

Where: $\beta_{h}$ is the sloping factor, $\theta_{j}^{r} \mathrm{~ns}$ is the number of levels or sigmoids in the hidden unit, and define the jump places in the transfer function.

\section{RESULTS}

In this research, we compared the WT, ICA, using optimized QNN utilizing EEG signals from normal and abnormal patients. Wavelet transform and ICA were used for feature extraction and the dimensions are then reduced. These data were used as inputs in a classification system based on improved QNN.The introduced recognition system is designed by using MATLAB 2020b, estimated with 5 - classes for the EEG signals. This section shows the results of experimental classification on the EEG data set, which is used in Optimized QNN by PSO. The obtained results by combining the ICA, WT, and two distinct scalp electrodes. as in Table $1-4$.

Table 1. Classification results using ICA an an optimized QNN no.1

\begin{tabular}{ccccccc}
\hline & Tp & Fn & Fp & Se & Pp & TNR \\
\hline Class 1 & 25 & 1 & 3 & 0.98 & 0.887 & 0.892 \\
Class 2 & 24 & 2 & 4 & 0.943 & 0.889 & 0.8571 \\
Class 3 & 24 & 2 & 3 & 0.922 & 0.866 & 0.888 \\
Class 4 & 25 & 2 & 2 & 0.856 & 0.857 & 0.9259 \\
Class 5 & 21 & 4 & 3 & 0.857 & 0.864 & 0.875 \\
TCA & & & $82.4 \%$ & & & \\
\hline
\end{tabular}

Table 2. Classification results using ICA an an optimized QNN no.5

\begin{tabular}{ccccccc}
\hline & Tp & Fn & Fp & Se & Pp & TNR \\
\hline Class 1 & 25 & 0 & 5 & 0.99 & 0.857 & 0.833 \\
Class 2 & 24 & 0 & 7 & 1 & 0.812 & 0.774 \\
Class 3 & 24 & 0 & 3 & 1 & 0.876 & 0.888 \\
Class 4 & 25 & 2 & 11 & 0.998 & 0.677 & 0.6944 \\
Class 5 & 21 & 0 & 7 & 1 & 0.764 & 0.75 \\
TCA & & & $78.43 \%$ & & & \\
\hline
\end{tabular}

Table 3. Classification results using WT an an optimized QNN no.1

\begin{tabular}{ccccccc}
\hline & Tp & Fn & Fp & Se & Pp & TNR \\
\hline Class 1 & 25 & 1 & 3 & 0.931 & 0.899 & 0.892 \\
Class 2 & 23 & 3 & 5 & 0.921 & 0.789 & 0.8214 \\
Class 3 & 22 & 3 & 3 & 0.897 & 0.876 & 0.88 \\
Class 4 & 25 & 2 & 4 & 0.932 & 0.865 & 0.8620 \\
Class 5 & 22 & 4 & 7 & 0.867 & 0.764 & 0.7586 \\
TCA & & & $77.8 \%$ & & & \\
\hline
\end{tabular}




\begin{tabular}{ccccccc}
\multicolumn{6}{c}{ Table 4. C Classification results using WT an an optimized QNN no.5 } \\
\hline & Tp & Fn & Fp & Se & Pp & TNR \\
\hline Class 1 & 24 & 0 & 6 & 1 & 0.847 & 0.82 \\
Class 2 & 24 & 2 & 5 & 0.896 & 0.862 & 0.8275 \\
Class 3 & 21 & 6 & 2 & 0.764 & 0.966 & 0.9130 \\
Class 4 & 23 & 4 & 3 & 0.828 & 0.887 & 0.8846 \\
Class 5 & 20 & 8 & 4 & 0.712 & 0.864 & 0.8333 \\
TCA & & & $75 . \%$ & & & \\
\hline
\end{tabular}

Where,Tpi is positively true classification for $i^{\text {th }}$ class and Fni is negatively false classification for ith class. Fpi is positively false classification for $\mathrm{i}^{\text {th }}$ class. The sensitivity which is also called true positive is defined as :

$$
\begin{aligned}
& S e=\frac{T p i}{T p i+F n i} \\
& \mathrm{Pp}=\frac{T p i}{T p i+F p i}
\end{aligned}
$$

Where Pp is the positive productivity. The specificity value (true negative) is derived by dividing the total number of diagnoses by the total number of diagnoses reported by the expert neurologists. The true negative ratio, also known as specificity, is determined using the formula:

$$
\mathrm{T}_{\mathrm{NR}}=\frac{T n i}{T n i+F p i}
$$

According to simulation results in Table $1-4$, the total classification for ICA is 82.4 percent, while the total classification for WT is 78.43 percent; from these results, using the ICA for feature extraction is better than using WT. Figure 1 below represents the simulation of the EEG signals and the difference between the normal EEG and the abnormal one.

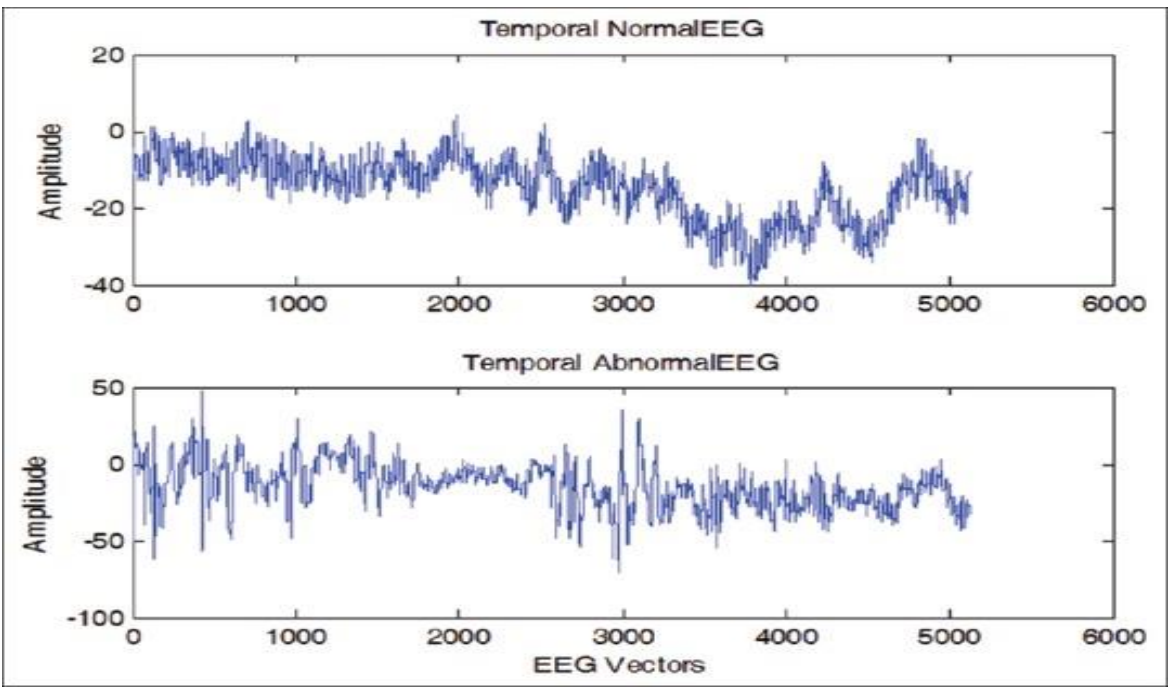

Figure 1. The normal and abnormal EEG signal

\section{DISSCUSION}

To circumvent this problem, improved QNN is used in this study. Second, in prior studies, the algorithms immediately sent all of classifiers collected features. However, there is a mixed distribution between classes in general due to a large variance in the EEG pattern distributions. However, the mixed distributions between the different classes in a general due to the large variance in the pattern distribution of the EEG. If a feature modification method that minimizes within-class scatter while maximizing scatter between classes is applied in the system. The size of the overlap zone between classes should be lowered greatly, and classification performance should be improved. To achieved this, the WT, ICA which are used in the proposed structures. Based on the simulation result for the proposed and experiences in the classification problems in the signals of EEG. I would like to make some of the following important points:

1. The enhanced QNN classifier's excellent classification accuracy provides insight into the features utilized to define EEG signals. The concluded applications of the coefficients of ICA are features that accurately reflect EEG signals, and that using the features, a better difference between the classes may be achieved as compared to WT. 
2. When compared to the ANN, the classification results and statistical parameter values showed that the optimized QNN with the ICA, WT had a lot of success in classifying EEG signals, the suggested combined ICA and WT approaches with optimized QNN approach can be evaluated.

3. The optimized QNN diagnostic system's testing performance has been determined to be satisfactory, and we believe that once developed this system has the potential to be employed in clinical. This tool makes evaluating EEG signals objective, and its automated nature makes it simple to utilize in clinical practice. Aside from the capability of a real-time deployment of the expert diagnostic system, increasing the diversity and quantity of parameters can improve diagnosis accuracy.

\section{CONCLUSION}

As a result of this study, several electrodes yielded various results and different mental tasks for various brain positions. The results from the first electrode were generally best from the fifth electrode, And the performance of the system by using ICA is better than WT in feature extraction of the EEG signals. Furthermore, class (4) classification from the first electrode is superior to class (5) from the fifth electrode. The simulation results show a high accuracy after QNN optimization by PSO. The optimized QNN provided us with somewhat faster and more realistic results. According to simulation results, the total classification for ICA is 82.4 percent, while the total classification for WT is 78.43 percent; from these results, using the ICA for feature extraction is better than using WT.

\section{REFERENCES}

[1] J. M. Kumar and V. K. Mittal, "EEG Data Acquisition System and Analysis of EEG Signals," 2021 2nd International Conference for Emerging Technology (INCET), 2021, pp. 1-5, doi: 10.1109/INCET51464.2021.9456431.

[2] M. Saini, U. Satija and M. D. Upadhayay, "Wavelet Based Waveform Distortion Measures for Assessment of Denoised EEG Quality With Reference to Noise-Free EEG Signal," in IEEE Signal Processing Letters, vol. 27, pp. 1260-1264, 2020, doi: 10.1109/LSP.2020.3006417.

[3] Wu, X. Y., S. H. Wang, and Y. D. Zhang. "Overview of k nearest neighbor algorithm theory and application." Computer Engineering and Application 53.21 (2017): 1-7..

[4] Hafeez Ullah Amin, Wajid Mumtaz, Ahmad Rauf Subhani, " Classification of EEG Signals Based on Pattern Recognition Approach", 21 November 2017, Department of Electrical and Electronic Engineering, Universiti Teknologi Petronas, Seri Iskandar, Malaysia.

[5] Dongmei Zhou and Xuemei Li," Epilepsy EEG Signal Classification Algorithm Based on Improved RBF", Chengdu University of Technology, Chengdu, , 23 June 2020, China.

[6] Jianguo Liu, Blake Woodson," Deep Learning Classification for Epilepsy Detection Using a Single Channel Electroencephalography (EEG)", Proceedings of the 2019 3rd International Conference on Deep Learning TechnologiesJuly 2019.

[7] Tahernezhad-Javazm, Farajollah, Vahid Azimirad, and Maryam Shoaran. "A review and experimental study on the application of classifiers and evolutionary algorithms in EEG-based brain-machine interface systems." Journal of neural engineering 15.2 (2018): 021007.

[8] Lu, Yao, Huiping Jiang, and Wenqiang Liu. "A review of EEG signal classifier based on deep learning." Proceedings of Information Science and Cloud Computing - PoS (ISCC 2017), Guangzhou, China (2018).

[9] Ömer Türk,Mehmet Siraç Özerdem,, "The convolutional neural network approach from electroencephalogram signals in emotional detection", Volume33, Issue20,07 May 2021.

[10] H. Donmez and N. Ozkurt, "Emotion Classification from EEG Signals in Convolutional Neural Networks," 2019 Innovations in Intelligent Systems and Applications Conference (ASYU), 2019, pp. 1-6, doi: 10.1109/ASYU48272.2019.8946364.

[11] C. L. Zhen, Random Noise Suppression of Seismic Signal Based on Wavelet Transform, Donghua University of technology, 2018.

[12] Chakole A.R., Barekar P.V., Ambulkar R.V., Kamble S.D. "(2019) Review of EEG Signal Classification". In: Satapathy S., Joshi A. (eds) Information and Communication Technology for Intelligent Systems. Smart Innovation, Systems and Technologies, vol 107. Springer, Singapore.

[13] Pengcheng Ma, Qian Gao, "EEG Signal and Feature Interaction Modeling-Based Eye Behavior Prediction Research", Computational and Mathematical Methods in Medicine, vol. 2020, Article ID 2801015, 10 pages, 2020. https://doi.org/10.1155/2020/2801015.

[14] R. Inoue, T. Sugi, Y. Matsuda, S. Goto, H. Nohira and R. Mase, "Recording and Characterization of EEGs by Using Wearable EEG Device," 2019 19th International Conference on Control, Automation and Systems (ICCAS), 2019, pp. 194-197, doi: 10.23919/ICCAS47443.2019.8971564.

[15] B. Nguyen, D. Nguyen, W. Ma and D. Tran, "Investigating the possibility of applying EEG lossy compression to EEG-based user authentication," 2017 International Joint Conference on Neural Networks (IJCNN), 2017, pp. 79-85, doi: 10.1109/IJCNN.2017.7965839.

[16] Fahimi, Fatemeh, et al. "Inter-subject transfer learning with an end-to-end deep convolutional neural network for EEG-based BCI." Journal of neural engineering 16.2 (2019): 026007. 
[17] Lopez, Juan Manuel, et al. "Open Source EEG Platform with Reconfigurable Features for Multiple-Scenarios." Indonesian Journal of Electrical Engineering and Informatics (IJEEI) 6.3 (2018): 253-264.

[18] Francis, William C., C. Umayal, and G. Kanimozhi. "Brain-Computer Interfacing for Wheelchair Control by Detecting Voluntary Eye Blinks." Indonesian Journal of Electrical Engineering and Informatics (IJEEI) 9.2 (2021): 521-537.

[19] Jawad, Ali Talib, et al. "Design of Adaptive Controller for Robot Arm Manipulator Based on ANN with Optimized PID by IWO Algorithm." 2021 International Conference on Advanced Computer Applications (ACA). IEEE, 2021.

[20] Abdulkarim, Haider, and Mohammed Z. Al-Faiz. "Online multiclass EEG feature extraction and recognition using modified convolutional neural network method." International Journal of Electrical and Computer Engineering (IJECE) 11.5 (2021): 4016-4026.

[21] Wang, Fei, et al. "Emotion recognition with convolutional neural network and EEG-based EFDMs." Neuropsychologia 146 (2020): 107506.

[22] George, Fabian Parsia, et al. "Recognition of emotional states using EEG signals based on time-frequency analysis and SVM classifier." International Journal of Electrical \& Computer Engineering (2088-8708) 9.2 (2019).

[23] M. A. Riyadi, I. Setiawan and A. Amir, "EEG Multiclass Signal Classification Based on Subtractive ClusteringANFIS and Wavelet Packet Decomposition," 2021 International Conference on Electrical and Information Technology (IEIT), 2021, pp. 81-86, doi: 10.1109/IEIT53149.2021.9587407.

[24] C. Y. Sai, N. Mokhtar, H. Arof, P. Cumming and M. Iwahashi, "Automated Classification and Removal of EEG Artifacts With SVM and Wavelet-ICA," in IEEE Journal of Biomedical and Health Informatics, vol. 22, no. 3, pp. 664-670, May 2018, doi: 10.1109/JBHI.2017.2723420.

[25] K. Tomonaga, S. Wakamizu and J. Kobayashi, "Experiments on classification of electroencephalography (EEG) signals in imagination of direction using a wireless portable EEG headset," 2015 15th International Conference on Control, Automation and Systems (ICCAS), 2015, pp. 1805-1810, doi: 10.1109/ICCAS.2015.7364652.

\section{BIOGRAPHY OF AUTHORS}

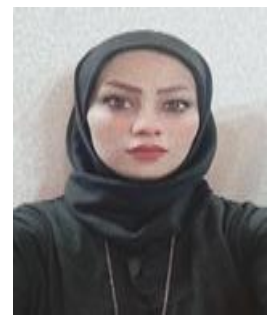

Dalael Saad Abdul-Zahra : received the B.Sc Mathematical from Babylon University, Babylon, Iraq, in 2015 and M.SC. Mathematical ( Factional Analysis) from Kufa University, Najaf, Iraq, in 2020 , I am currently working at Hilla University College as an assistant teacher in the Department of Medical Physics Interested in studying topics related to applied and pure mathematics, rubber engineering and some special interests in computers and basic programming languages . He can be contacted at email: dalael20175@gmail.com .

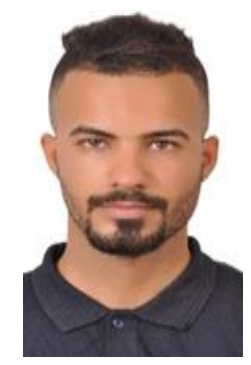

Ali Talib Jawad : received the B.Sc. and M.Sc. degrees in electrical engineering from Babylon University, Babylon, Iraq, in 2015 and 2018, respectively. He is currently a lecturer at Hilla University College, Department. Of Medical Instrumentation Technologies Engineering Babylon, Iraq. His research interests include the applications of artificial intelligence, control system, communications, the biomedical field, and robotics. He can be contacted at email: alitaleb54qq@gmail.com . 